\title{
The ITS Application of Mobile Phones to Solve the Signalized Intersections' Problems in Suburb of Bangkok, Thailand
}

\author{
Weeradej Cheewapattananuwong ${ }^{1}$, Sanit Srisuk ${ }^{2}$ \\ ${ }^{1}$ Director, Route Survey Division, Rural Roads Department, Ministry of Transport \\ 9 Paholyothin Rd, Bangkhen, Bangkok, Thailand \\ weeradej146@drr.go.th \\ ${ }^{2}$ Director, Bureau of Location and Design, Rural Roads Department, Ministry of Transport \\ 9 Paholyothin Rd, Bangkhen, Bangkok, Thailand \\ sanitsri@drr.go.th
}

\begin{abstract}
The Application of Intelligent Transportation Systems (ITS) of Rural Roads Department in Thailand have been developed more than 15 years. In this project, the mitigation of traffic signalization system is proposed for two intersections as applied for 3 legs and 4 legs intersections respectively. The collected traffic data that, were used by manual traffic counts, were compared with the image sensing software's data. These data were corrected and predicted for the fine tuning of traffic signalization under the statistic methods which were the Gamma Distribution with Density, Bootstrapping for directly generating inputting data and the Method of Maximum Likelihood (ML) to estimate the parameters. At the moment, traffic signalization system of two intersections cannot be synchronized by the cable network. However, it can be solved problems and remedied traffic situations by using of Mobile Phones' Network with the Private Cloud Service (PCS). There are three I-Cloud Servers which are VDO Collector, VDO Processor and Application Server. In addition, the calculation of traffic density at each leg of intersections is taken into consideration. This means that using of image sensing software under Lucas-Kanade Method is proposed for the reducing of processing times. In case of traffic density, the tracking of each vehicle-group within 40 meters of the first range will be shown the groups of queue lengths and the average speed of vehicles. Moreover, these factors from 40 meters to the out of range of tickers under the image sensing software from CCTVs will be also taken into account. The effective traffic cycle times (ETCT) will decrease of the queue length and increase of the speed-vehicles by the skips of phasing within the optimization of traffic cycle times (OTCT). Finally, traffic policemen at the traffic controllers will change the phases promptly so as to remedy the traffic situations.
\end{abstract}

Keywords: Intelligent Transportation Systems (ITS), Gamma Distribution with Density, Bootstrapping, the Method of Maximum Likelihood (ML), Private Cloud Service (PCS), I-Cloud Servers, the Effective Traffic Cycle Times (ETCT), the Optimization of Traffic Cycle Times (OTCT).

\section{Introduction}

The traffic signalization system at two intersections are proposed for 3 legs and 4 legs intersections. The collected traffic data by the manual traffic counts are evaluated with the image sensing software's counting. These data are corrected, calibrated and predicted by the fine tuning of traffic signalization under the statistic methods. The Gamma Distribution with Density, Bootstrapping for directly generating inputting data and the Method of Maximum Likelihood (ML) to estimate the parameters are taken into account. The length of both intersections in this case is more than 500 meters therefore, the effectiveness of cable-signal cannot solve the traffic problems as the Mobile Phones' Network System. The Private Cloud Service (PCS) such as, I-Cloud Services is proposed for the 3 sources (24 hours) which are VDO Collector - compressed from CCTVs, VDO Processor-tracked motions of vehicles and Application Server-evaluated vehicle-density. The calculations of traffic density of each leg-intersections are located within the 40 meters in length of the first range. The second range-from 40 meters to the end of CCTVs-Sight is assessed of the density by the image sensing software. The statistical method such as Lucas-Kanade process [1] is proposed for the reducing of processing times. In case of traffic density, the tracking of each vehicle-group within 40 meters of the first range is shown the queue lengths (19 vehicles) and the average speed of vehicles $(<40 \mathrm{~km} / \mathrm{hr})$ as illustrated in the collected data. Moreover, these traffic factors from 40 meters to the out of range of tickers under the image sensing software from CCTVs are also 
evaluated as the mentioned process. The vehicle actuated (VA) concept is taken into account so as to decrease the cycle times or loss times periods or the queue length within the effective traffic cycle times (ETCT) and to skip phasing for the increasing of the speed-vehicles within the optimization of traffic cycle times (OTCT) as shown in this processes. This method under the iOS- System is compared with the Google Traffic Report Map under the Android System however, the compared data are different from the real time situations. For examples, a traffic accident in the first area had an effect on road users when a traffic policeman came to remedy this situation after more alerts from his walky-talky. Although, the other policeman used the Google Map-Application, it leaded to the long queues of intersections (at least 200 meters). Therefore, this application can help the policemen promptly to solve and mitigate the traffic problems at the real times.

\section{Criteria of Traffic Data Collection}

The concepts of collected traffic data at two intersections in the suburb of Bangkok, Thailand are proposed for uses of manual method and image sensing software's method as illustrated in the Figure1.

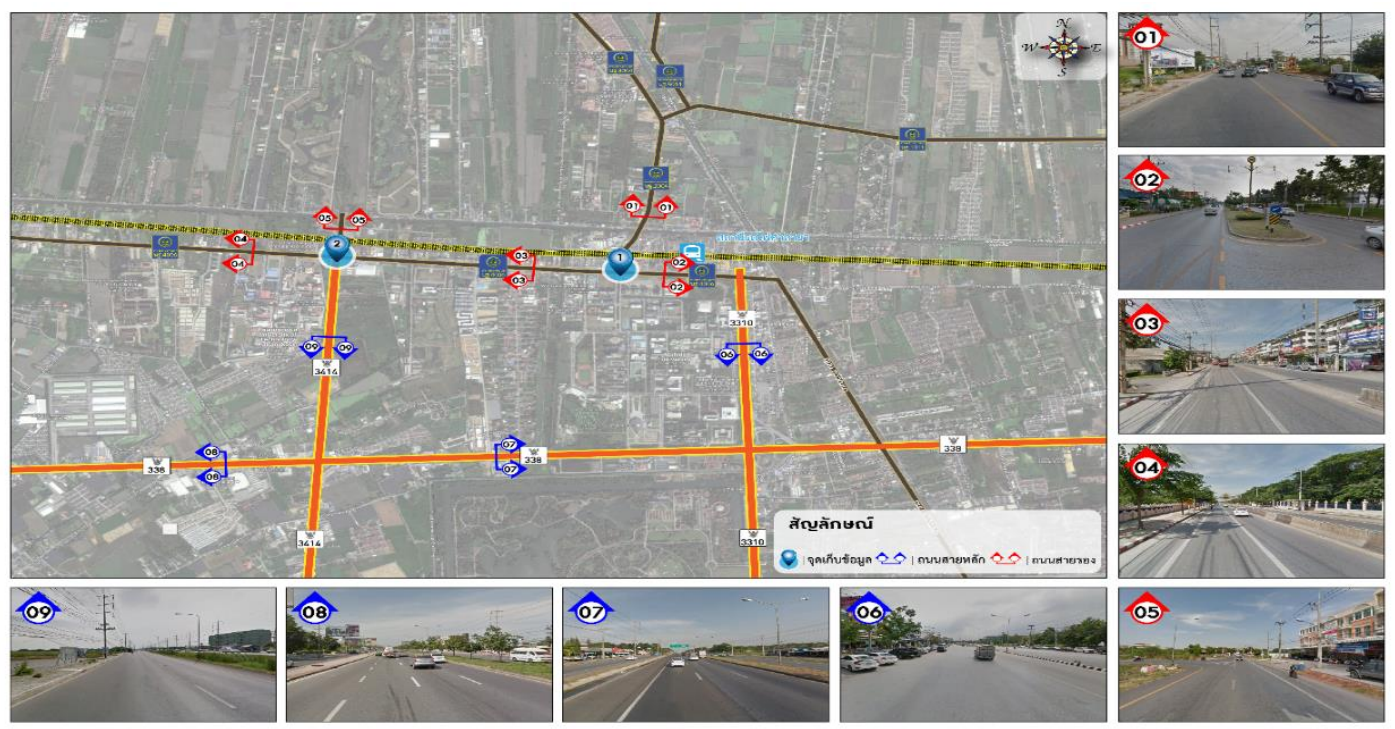

Fig. 1: Location of Two Intersections in the Suburb of Bangkok.

In case of Image Sensing Software, the researchers applies the Analysis of Pixels under the Mask and Vehicle Classification techniques by the Ensemble Learning Method. In case of the mask evaluation under Lucas-Kanade method, this process leads to the best solution to classify the types of vehicles in the short times. The moving of each pixel $(d x, d y)$ at the time $d t$ is compared with the pixels at the starting location as shown in the following equations under the Taylor Series.

$$
\begin{gathered}
I(x, y)=I(x+d x, y+d y, d t) \\
I(x+d x, y+d y, d t) \rightarrow f_{x} u+f_{y} v+f_{t}=0 \\
f_{x}=\frac{\partial f}{\partial x}, f_{y}=\frac{\partial f}{\partial y}, u=\frac{d x}{d t}, v=\frac{d y}{d t}
\end{gathered}
$$

and unknown variables, $u$ and $v$

$$
\left[\begin{array}{l}
u \\
v
\end{array}\right]=\left[\begin{array}{cc}
\sum_{i} f_{x_{i}}^{2} & \sum_{i} f_{x_{i}} f_{y_{i}} \\
\sum_{i} f_{x_{i}} f_{y_{i}} & \sum_{i} f_{y_{i}}^{2}
\end{array}\right]^{-1}\left[\begin{array}{l}
-\sum_{i} f_{x_{i}} f_{t_{i}} \\
-\sum_{i} f_{y_{i}} f_{t_{i}}
\end{array}\right]
$$




$$
v_{p_{i}}=\frac{\sqrt{\left(x_{i_{2}}-x_{i_{1}}\right)^{2}+\left(y_{i_{2}}-y_{i_{1}}\right)^{2}}}{\left(t_{2}-t_{1}\right)}
$$

Tracking Data are distinguished from the Decision Boundary-Generated Method by Individual Classifiers as shown in the Observation and Measurement-Figure (Fig. 2). As can be seen that the Decision Boundary-Generated Method is classified the group of same data in the circle clusters. The vehicle data in the each direction are evaluated in the different lengths of Angle-Sights from CCTVs. For examples, the 26 and 37 meters of angle-sight lengths are depended from the focuses of optical lens in order to calibrate with the manual measurements.

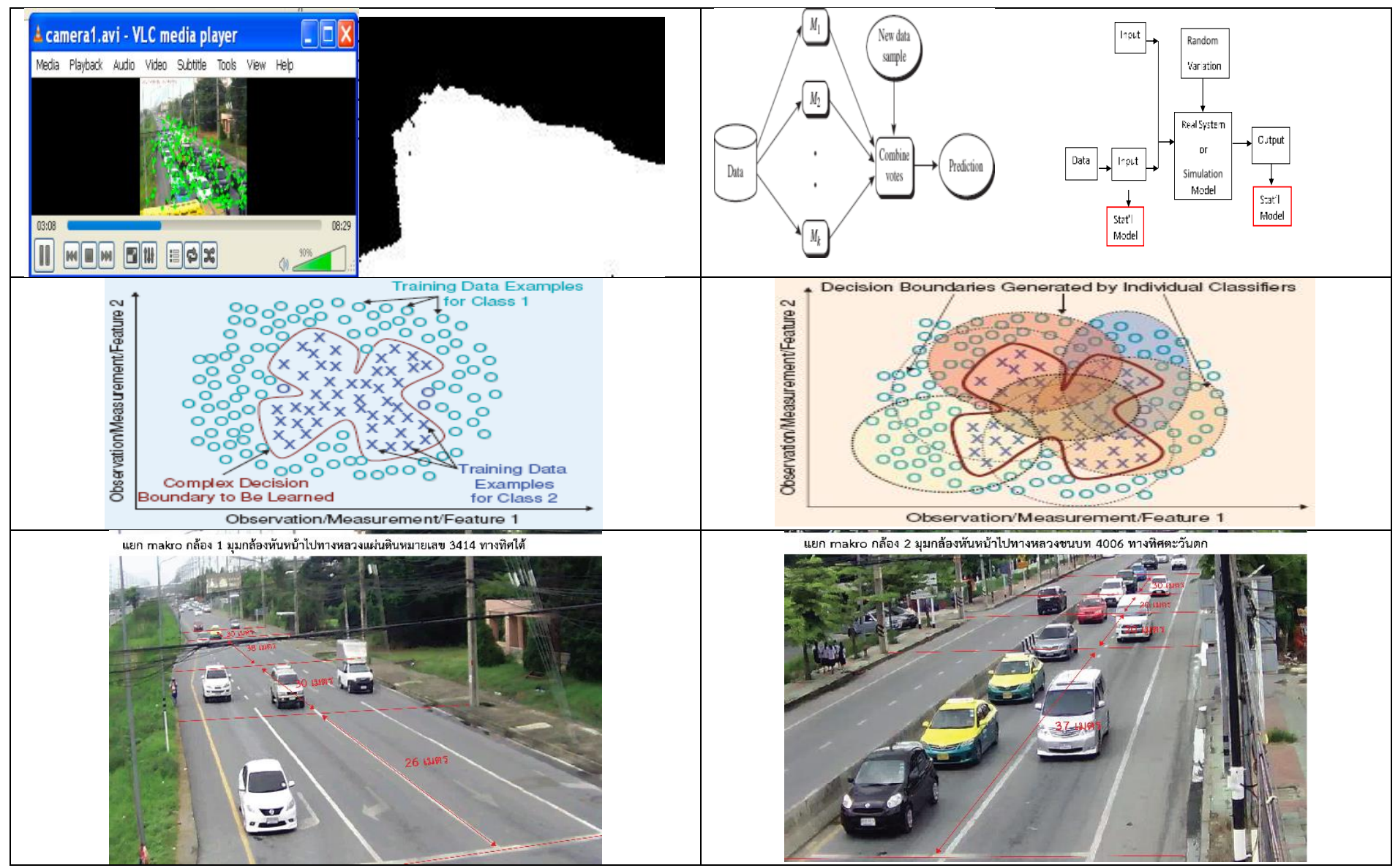

Fig. 2: Vehicle Classification of the Image Sensing software by the Ensemble Learning Method.

\section{Analysis of Collected Data by Statistical Method}

In a simulation study, the operation of traffic signalization supposed that the interest was in estimating the average waiting time of vehicles at the intersection. The inter-arrival times were to be modelled as exponentially distributed random variables with a given arrival rate $\lambda$. The gamma distribution with density function was to be used as the model of service times. $C$, the number of green times was set to just one. In this case, the researchers would treat $\lambda$ and $C$ as input variables of type $X$, whilst $\alpha$ and $\beta$ were regarded as input parameters of type $\theta$ [2].

$$
f_{G}(s ; \alpha, \beta)=\frac{s^{\alpha-1} \exp (-s / \beta)}{\Gamma(\alpha) \beta^{\alpha}}, s>0
$$




\subsection{In Case of 3 Legs Intersection}

Input Modelling Example: In the Traffic Signalization Example, the researchers had already selected the gamma distribution with density as the service time distribution. In term of the parameters, $\alpha$ and $\beta$ of the distribution were unknown, but that researchers had some existing real service time or effective green times-data, s. The problem then was to fit the gamma distribution by estimating $\alpha$ and $\beta$ from this data. The alternative bootstrapping was used directly for generating inputs and the method of maximum likelihood (ML), to estimate the parameters was taken into account. The Traffic Volumes-Data of 3 Legs Intersection with 90 seconds of a cycle times were collected within 24 hours in 3 months as shown in Table1.

Table 1: Traffic Volumes Data (PCU per Hour) of 3 Legs Intersection within 3 months at the 90 seconds of cycle times.

\begin{tabular}{|c|c|c|c|c|c|c|c|}
\hline \multirow[b]{2}{*}{ Directional } & \multirow{2}{*}{$\begin{array}{c}\mathrm{MIN} \\
(\mathrm{Pcu} / \mathrm{Hr})\end{array}$} & \multirow{2}{*}{$\begin{array}{c}\text { MAX } \\
(\mathrm{Pcu} / \mathrm{Hr})\end{array}$} & \multirow[b]{2}{*}{ Traffic Volume (Pcu/Hr) } & \multirow[b]{2}{*}{ PCU/MIN } & \multirow[b]{2}{*}{ PCU/SEC } & Density $<40 \mathrm{~m}$ & Density $>40 \mathrm{~m}$ \\
\hline & & & & & & PCU/MIN & PCU/MIN \\
\hline e-w & 492.53 & $1,050.94$ & 972.00 & 16.20 & 0.27 & 24.30 & 40.50 \\
\hline w-e & 550.59 & $1,109.67$ & 890.00 & 14.83 & 0.25 & 22.25 & 37.08 \\
\hline w-n & 318.19 & 971.70 & 917.00 & 15.28 & 0.25 & 22.93 & 38.21 \\
\hline e-n & 571.68 & $1,284.67$ & 683.00 & 11.38 & 0.19 & 17.08 & 28.46 \\
\hline$n-e$ & 632.00 & $1,044.54$ & 722.00 & 12.03 & 0.20 & 18.05 & 30.08 \\
\hline n-w & 383.42 & $1,577.61$ & 712.00 & 11.87 & 0.20 & 17.80 & 29.67 \\
\hline
\end{tabular}

The Fitting by Gamma Distribution of the estimating $\alpha$ and $\beta$ from these data were presented in the following table. Furthermore, the alternative bootstrapping was used directly for generating inputs and the method of maximum likelihood (ML) was also shown the Table 2.

Table 2: The Estimation of Parameters by ML Method.

\begin{tabular}{|c|c|c|c|c|}
\hline & Initial Values & ML Estimates & $\begin{array}{c}\text { Lower 95\% } \\
\text { Limit }\end{array}$ & $\begin{array}{c}\text { Upper 95\% } \\
\text { Limit }\end{array}$ \\
\hline Parameters & 17.2702694 & 17.2702694 & -2.114369341 & 36.65490815 \\
\hline b1 & 52.58759644 & 52.58759644 & -7.305151158 & 112.480344 \\
\hline
\end{tabular}

Therefore, the ML estimates were $\hat{\alpha}=17.27$ and $\hat{\beta}=52.59$ for these data set.

Given a random sample of observations $Y_{i}$ for $i=1,2, \ldots \mathrm{n}$, the empirical distribution function (EDF) was defined as:

$$
\tilde{F}(y)=\frac{\# \text { of } Y^{\prime} s \leq y}{n}
$$

Thus, Examples of EDF appeared in Figures 3. An EDF test statistic tests if a sample had been drawn from the distribution with $\operatorname{CDF} F_{0}(y)$, by looking at the difference between its $\operatorname{EDF} \tilde{F}(y)$ and $F_{0}(y)$. 


$$
T=\int \psi(y)\left(\tilde{F}(y)-F_{0}(y)\right)^{2} d F_{0}(y)
$$

Here, $\psi(y)$ was a weighting function. The true values of $\alpha$ and $\beta$ should be incorporated into the analysis of the output of the simulation model and the use of bootstrapping (Scatterplot of 500 bootstrap $\alpha_{i}^{*}$ and $\beta_{i}^{*}$, Input Modelling) was possible resolved as presented in the following Figure.

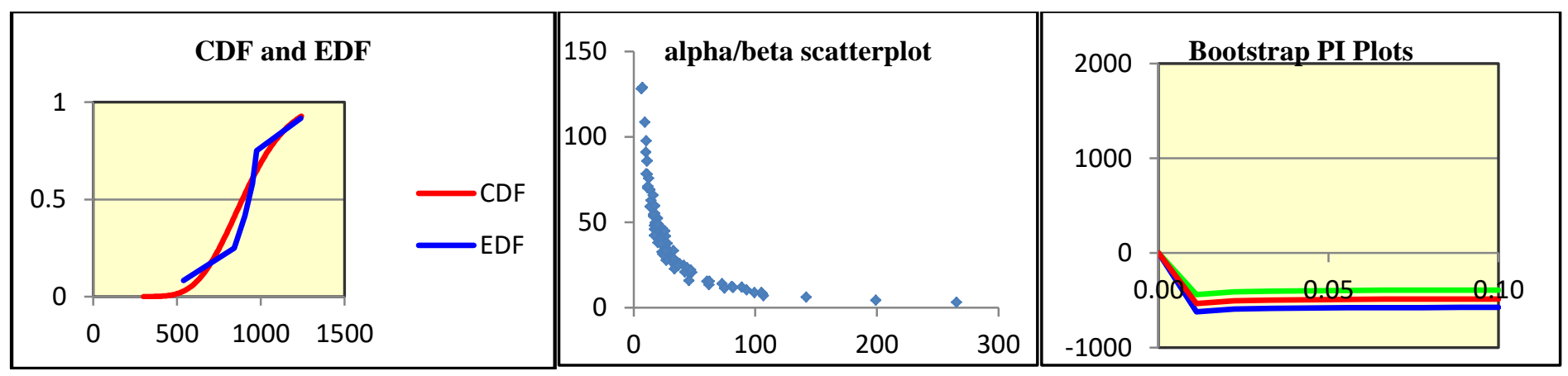

Fig. 3: Compared the CDF and EDF and Scatterplot of 500 bootstrap $\alpha_{i}^{*}$ and $\beta_{i}^{*}$, Input Modelling at 3 Legs Intersection.

\subsection{In Case of 4 Legs Intersection}

The next Table showed the Traffic Data at 4 Legs Intersection with 120 seconds of cycle times were collected within 24 hours in 3 months.

Table 2: Traffic Volumes Data (PCU per Hour) of 4 Legs Intersection within 3 months at the 120 seconds of cycle times.

\begin{tabular}{|c|c|c|c|c|c|c|c|}
\hline & MIN & MAX & & & & Density $<40 \mathrm{~m}$ & Density $>40 \mathrm{~m}$ \\
\hline Dirctional & $(\mathrm{Pcu} / \mathrm{Hr})$ & $(\mathrm{Pcu} / \mathrm{Hr})$ & Traffic Volume $(\mathrm{Pcu} / \mathrm{Hr})$ & PCU/MIN & PCU/SEC & PCU/MIN & PCU/MIN \\
\hline e-w & 424.89 & $1,524.31$ & 816.00 & 13.60 & 0.23 & 27.20 & 40.8 \\
\hline e-n & 82.69 & 351.14 & 122.00 & 2.03 & 0.03 & 4.07 & 6.1 \\
\hline e-s & 215.34 & 976.51 & 718.00 & 11.97 & 0.20 & 23.93 & 35.9 \\
\hline w-e & 319.14 & 721.34 & 370.00 & 6.17 & 0.10 & 12.33 & 18.5 \\
\hline $\mathrm{w}-\mathrm{n}$ & 140.07 & 342.42 & 306.00 & 5.10 & 0.09 & 10.20 & 15.3 \\
\hline $\mathrm{w}-\mathrm{s}$ & 434.98 & 683.32 & 626.00 & 10.43 & 0.17 & 20.87 & 31.3 \\
\hline n-e & 197.54 & 372.81 & 288.00 & 4.80 & 0.08 & 9.60 & 14.4 \\
\hline $\mathrm{n}-\mathrm{w}$ & 178.79 & 260.10 & 254.00 & 4.23 & 0.07 & 8.47 & 12.7 \\
\hline $\mathrm{n}-\mathrm{s}$ & 301.32 & 765.20 & 659.00 & 10.98 & 0.18 & 21.97 & 32.95 \\
\hline$s-n$ & 276.12 & 534.88 & 415.00 & 6.92 & 0.12 & 13.83 & 20.75 \\
\hline s-w & 346.76 & 708.96 & 627.00 & 10.45 & 0.17 & 20.90 & 31.35 \\
\hline s-e & 450.78 & 981.53 & 914.00 & 15.23 & 0.25 & 30.47 & 45.7 \\
\hline
\end{tabular}


The Fitting by Gamma Distribution of the estimating $\alpha$ and $\beta$ from these data were also presented in the Table 3 . Moreover, the alternative bootstrapping was proposed and the ML was illustrated in the Table 3.

Table 3: The Estimation of Parameters by ML Method.

\begin{tabular}{|c|c|c|c|c|}
\hline Parameters & Initial Values & ML Estimates & $\begin{array}{c}\text { Lower 95\% } \\
\text { Limit } \\
\end{array}$ & $\begin{array}{l}\text { Upper 95\% } \\
\text { Limit } \\
\end{array}$ \\
\hline & 3.23732 & & 0.764337 & 5.710306 \\
\hline b1 & 191 & 3.23732191 & 662 & 159 \\
\hline b2 & $\begin{array}{r}160.421 \\
1154\end{array}$ & 160.4211154 & $\begin{array}{r}27.82208 \\
254\end{array}$ & $\begin{array}{r}293.0201 \\
483\end{array}$ \\
\hline
\end{tabular}

Thus, The ML estimates were $\hat{\alpha}=3.24$ and $\hat{\beta}=160.42$ for this data set.

EDF and EDF test statistic tests had been drawn from the distribution with CDF $F_{0}(y)$ and the true values of $\alpha$ and $\beta$ were an analysis of the output of the simulation model and the use of bootstrapping was resolved as presented in the Figure 4.

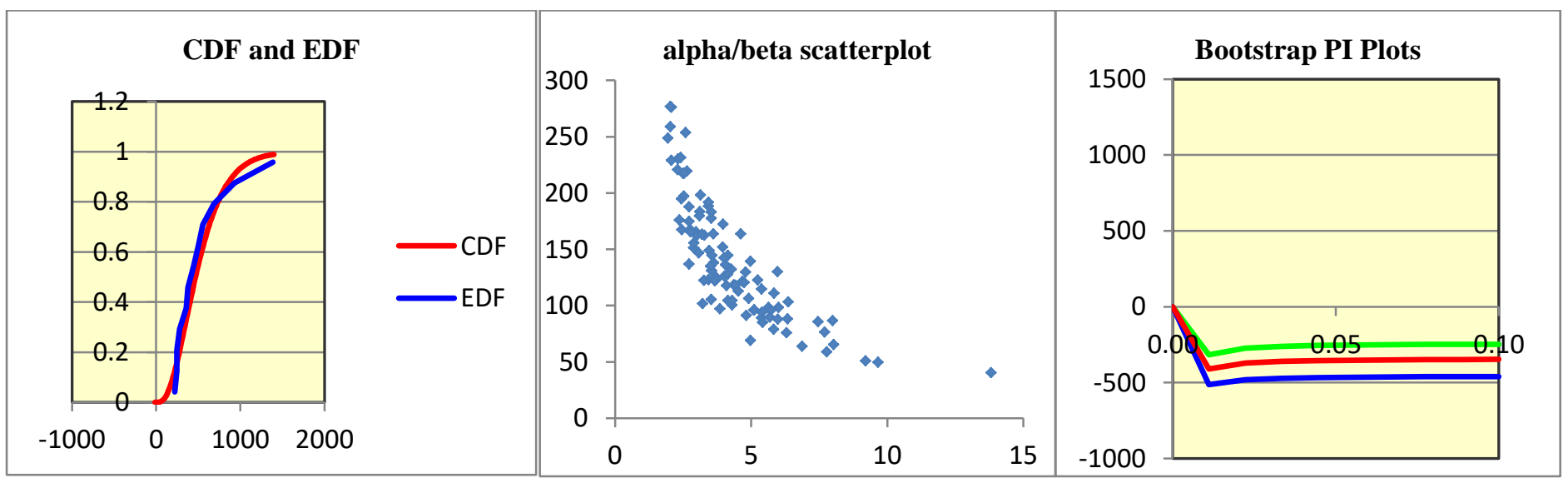

Fig. 4: Compared the CDF and EDF and Scatterplot of 500 bootstrap $\alpha_{i}^{*}$ and $\beta_{i}^{*}$, Input Modelling at 4 Legs Intersection.

\section{Evaluation of Traffic Signalization Factors Data} follows:

In case of Traffic Factors [3], Average Spacing $M$ and the Average Length of Changing Lane $L$ were described as

$$
M=\max \left[0.454652+\frac{0.269666 \times v}{\operatorname{atan}(\lambda)}, 8.20255\right]
$$

Where $\lambda$ was the arrival rate defined as $(\mathrm{PCU} / \mathrm{sec})$. The correlation coefficients for the equations were 1.00, 0.997, 0.997, and 0.995 , respectively.

Traffic data collected since 2017 have been evaluated by statistical theories; the fitted curve model and equations are as shown below. The Average Speed $v(\mathrm{kph})$ and the Effective Green Time $g_{e}$ (seconds) are obtained by the following equations:

$$
v=1.16115 \times \lambda-\frac{1.36164}{\cos (125.776 \lambda)}
$$




$$
g_{e}=12.7998 \times\left(52.923 \times e^{0.0056 \lambda}\right)-635.59
$$

Where $\lambda$ is the arrival rate defined as (PCU/min). The correlation coefficients for both the equations were 0.901 and 0.799 , respectively.

\section{Results of Traffic Model}

The Intersection 1 from Figure1 is shown the traffic situation form the Google Map. It is clearly seen in Figure 5 that, the maximum of queue length is more than 500 meters. In addition, the Figure 6 is illustrated of the queue length from CCTVs (number 05).
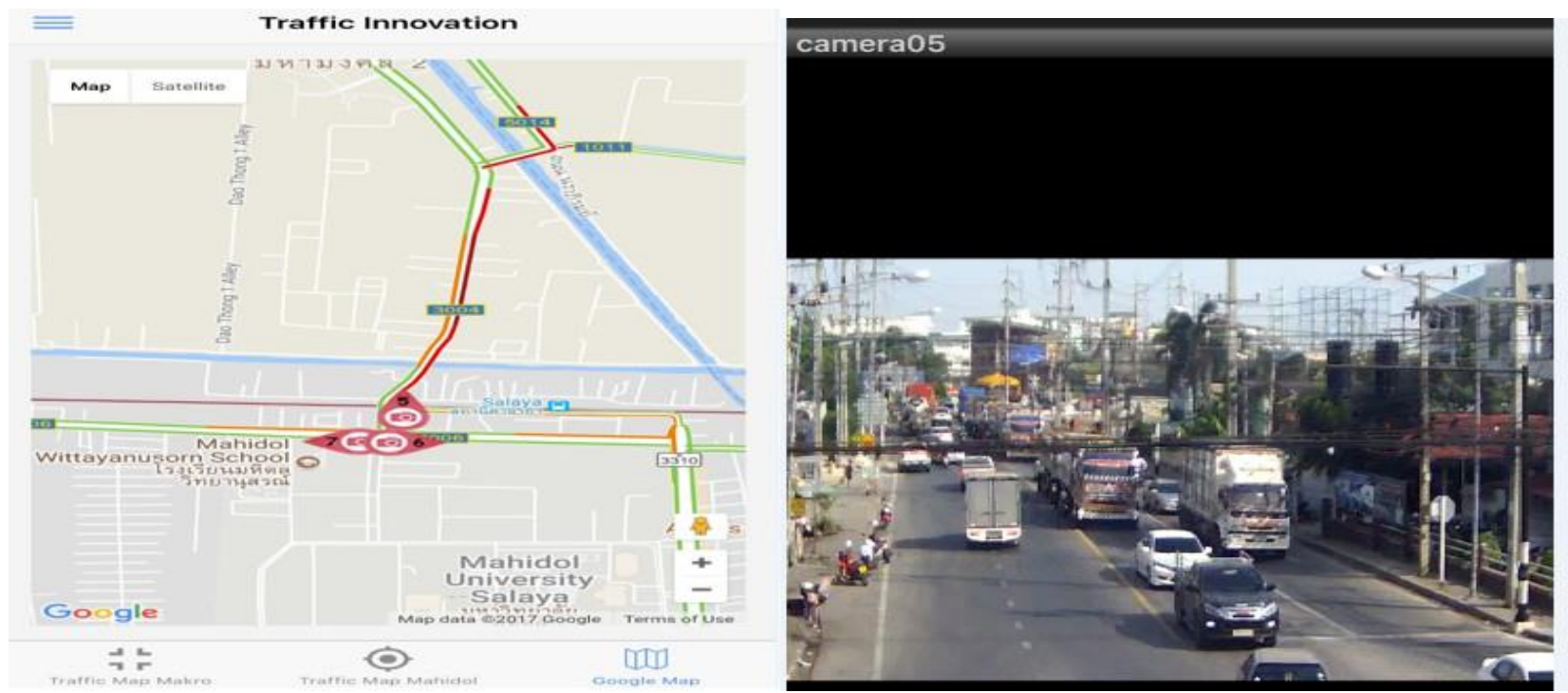

Fig. 5: the queue length compared between a Google Map and Real Times CCTVs.

The movement of pixels or vehicles under the traffic signals or each phasings and accumulation of queue lengths are presented at 09:12:00 am. The traffic situation at this time is somewhat congestion when compared with the 09:14:00 am. After 09:13:00, the speed of vehicles within 40 metres at intersection increase drastically and queue length is lower than 150 meters as shown in the Figure 6. 


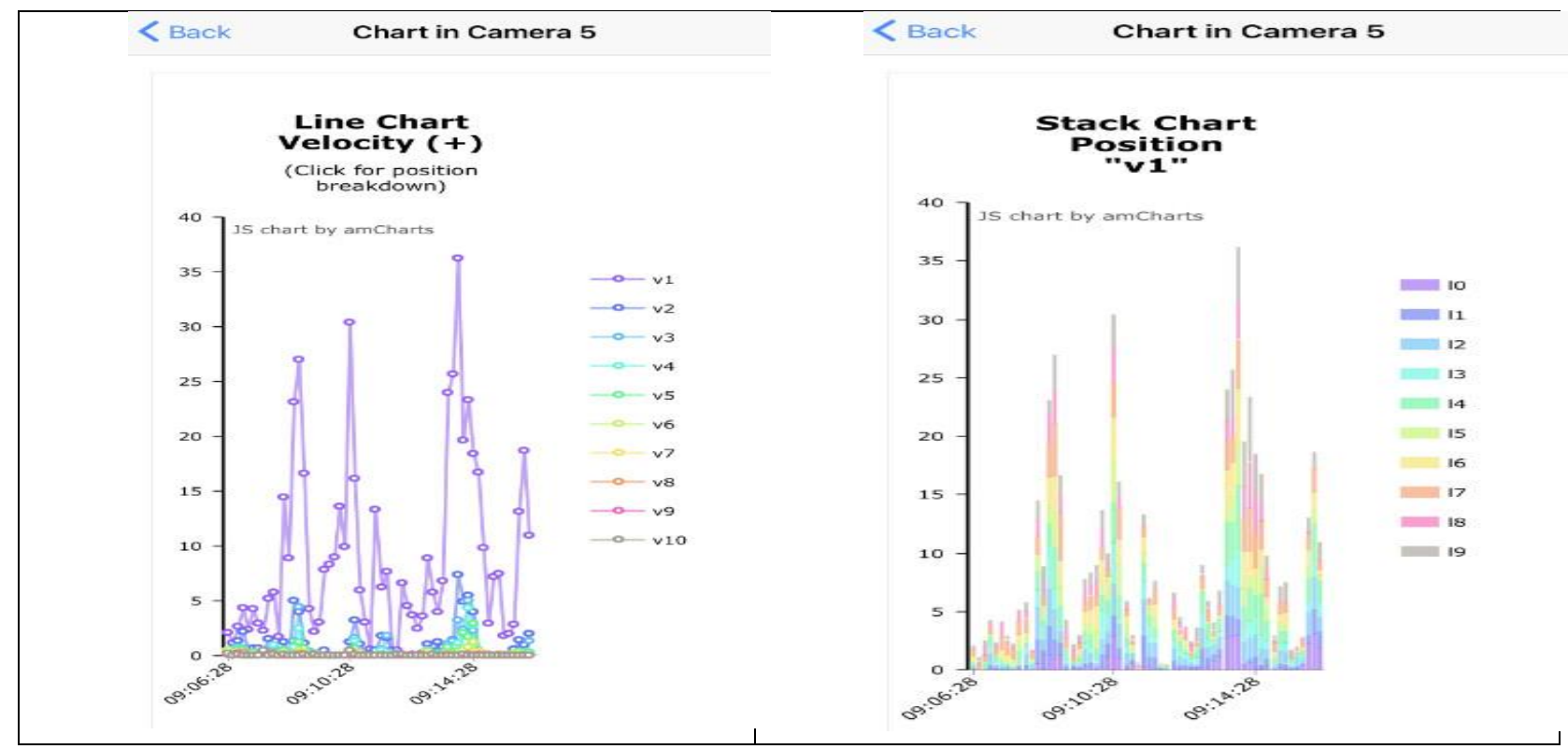

Fig. 6: Result of Vehicle-Speed at the different phashing of cycle times.

Finally, the Recommendation of Phasing and Cycle Times from the image sensing software is porposed on the Mobile Phone. Therefore, Policemen at the control room will be changed of the traffic phases and the effective green times based on these recommendation as shown in the Figure 7.

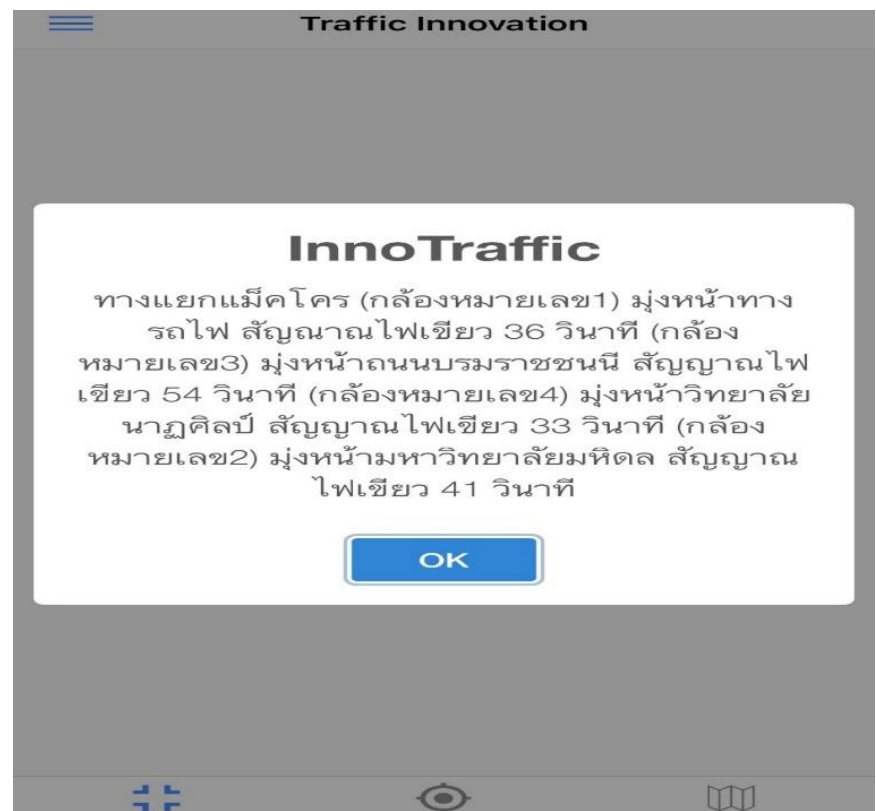

Fig. 7: Recommendation of Traffic Phasing and Effective Green Times.

\section{Conclusion}

This application is very useful for traffic policemen to control traffic controllers at the intersections. The traffic factors, such as speed and queue length results are better than the previous traffic situations. In the near future, the development of these methods will be linked and controlled by the Mobile Phones. Moreover, the security of software and data protections will be taken into consideration by the preventing cyber-attacks of gateway and i-Cloud. 


\section{Acknowledgements}

The researchers would like to thank Mr. Suporn Taechaiya, Chief Engineer in the Road Design so as to support and encourage our research.

\section{References}

[1] OpenCV 3.3.1. Open Source Computer Vision. [Online]. Available: https://docs.opencv.org/3.3.1/d7/d8b/tutorial py lucas kanade.html

[2] R. C. H. Cheng, "Validity and Comparing Simulation Models Using Resampling," Journal of Simulation, vol.1, no. 1, pp. 53-63, University of Southampton, Southampton, SO17 1NQ, 2006.

[3] W. Cheewapattananuwong, P. Taneerananon, T. Nakatsuji, Innovative ITS Technologies for Reducing Traffic Impacts at Bangkok Intersections, http://library.jsce.or.jp/jsce/open/00039/201211_no46/pdf/P63.pdf 